Washington University School of Medicine Digital Commons@Becker

Open Access Publications

6-7-2021

\title{
Soluble P-tau217 reflects amyloid and tau pathology and mediates the association of amyloid with tau
}

\author{
Niklas Mattsson-Carlgren \\ Lund University \\ Shorena Janelidze \\ Lund University \\ Randall J Bateman \\ Washington University School of Medicine in St. Louis \\ Ruben Smith \\ Lund University \\ Erik Stomrud \\ Lund University
}

See next page for additional authors

Follow this and additional works at: https://digitalcommons.wustl.edu/open_access_pubs

Please let us know how this document benefits you.

\section{Recommended Citation}

Mattsson-Carlgren, Niklas; Janelidze, Shorena; Bateman, Randall J; Smith, Ruben; Stomrud, Erik; Serrano, Geidy E; Reiman, Eric M; Palmqvist, Sebastian; Dage, Jeffrey L; Beach, Thomas G; and Hansson, Oskar, "Soluble P-tau217 reflects amyloid and tau pathology and mediates the association of amyloid with tau." EMBO Molecular Medicine. 13, 6. e14022 (2021).

https://digitalcommons.wustl.edu/open_access_pubs/10738

This Open Access Publication is brought to you for free and open access by Digital Commons@Becker. It has been accepted for inclusion in Open Access Publications by an authorized administrator of Digital Commons@Becker. For more information, please contact vanam@wustl.edu. 


\section{Authors}

Niklas Mattsson-Carlgren, Shorena Janelidze, Randall J Bateman, Ruben Smith, Erik Stomrud, Geidy E Serrano, Eric M Reiman, Sebastian Palmqvist, Jeffrey L Dage, Thomas G Beach, and Oskar Hansson 


\title{
Soluble P-tau217 reflects amyloid and tau pathology and mediates the association of amyloid with tau
}

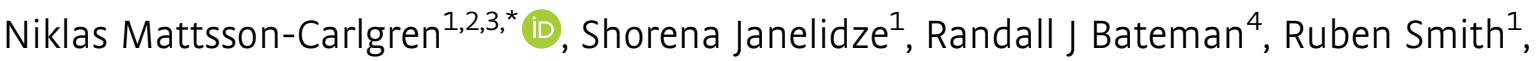 \\ Erik Stomrud $^{1,5}$, Geidy E Serrano ${ }^{6}$, Eric M Reiman ${ }^{6}$, Sebastian Palmqvist ${ }^{1,5}$ iD, Jeffrey L Dage ${ }^{7}$, \\ Thomas G Beach ${ }^{8}$ \& Oskar Hansson ${ }^{1,4,5},{ }^{* *}$ iD
}

\begin{abstract}
Alzheimer's disease is characterized by $\beta$-amyloid plaques and tau tangles. Plasma levels of phospho-tau217 (P-tau217) accurately differentiate Alzheimer's disease dementia from other dementias, but it is unclear to what degree this reflects $\beta$-amyloid plaque accumulation, tau tangle accumulation, or both. In a cohort with post-mortem neuropathological data $(N=88)$, both plaque and tangle density contributed independently to higher P-tau217, but P-tau217 was not elevated in patients with non-Alzheimer's disease tauopathies $(N=9)$. Several findings were replicated in a cohort with PET imaging ("BioFINDER-2", $N=426$ ), where $\beta$ amyloid and tau PET were independently associated with $\mathrm{P}$ tau217. P-tau217 concentrations correlated with $\beta$-amyloid PET (but not tau PET) in early disease stages and with both $\beta$-amyloid and (more strongly) tau PET in late disease stages. Finally, Ptau217 mediated the association between $\beta$-amyloid and tau in both cohorts, especially for tau outside of the medial temporal lobe. These findings support the hypothesis that plasma P-tau217 concentration is increased by both $\beta$-amyloid plaques and tau tangles and is congruent with the hypothesis that P-tau is involved in $\beta$-amyloid-dependent formation of neocortical tau tangles.
\end{abstract}

Keywords Alzheimer's disease; amyloid; phosphorylated tau; plasma; tau Subject Categories Biomarkers; Neuroscience DOI 10.15252/emmm.202114022 | Received 26 January 2021 | Revised 18 March 2021 | Accepted 23 March 2021 | Published online 5 May 2021 EMBO Mol Med (2021) 13: e14022

\section{Introduction}

$\beta$-amyloid (A $\beta$ ) pathology and tau pathology are core hallmarks of Alzheimer's disease (AD). The amyloid cascade hypothesis states that altered $A \beta$ metabolism is an initiating event, which drives accumulation of aggregated tau (Sperling et al, 2014). These events are associated with altered levels of fluid biomarkers, as has been extensively studied in both cerebrospinal fluid (CSF) and plasma (Olsson et al, 2016; Hampel et al, 2018). Recently, it has been shown that plasma levels of hyperphosphorylated tau (including both P-tau181 (Janelidze et al, 2020; Karikari et al, 2020) and P-tau217 (Palmqvist et al, 2020)) are markedly elevated in patients with AD. Plasma Ptau217 levels have particularly high accuracy for AD and are correlated with tangle densities in post-mortem data (Palmqvist et al, 2020). However, recent studies have suggested that CSF P-tau217 (and other P-tau variants) can be increased as a function of $A \beta$ accumulation (Suárez-Calvet et al, 2020), also in individuals who are tau-negative as determined by positron emission tomography (PET) (Barthélemy et al, 2020b; Mattsson-Carlgren et al, 2020) (but note that tau PET has limited sensitivity to detect very sparse tau aggregation (Fleisher et al, 2020)). It is unclear to what degree plasma Ptau217 levels depend on $A \beta$ versus tau load in the brain. Here, we set out to clarify this question in two cohorts, including a cohort with post-mortem quantification of $A \beta$ plaques and tau tangles, and a large prospective cohort where $A \beta$ and tau load were measured in vivo using PET imaging. In sum, we found converging evidence from both cohorts that $A \beta$ and tau load had independent and interactive effects on plasma P-tau217. We also found that plasma Ptau217 levels statistically mediated the effect of $A \beta$ load on tau load. Finally, we found that in subjects with very limited tangle pathology, $A \beta$ plaque load but not medial temporal lobe tangle load

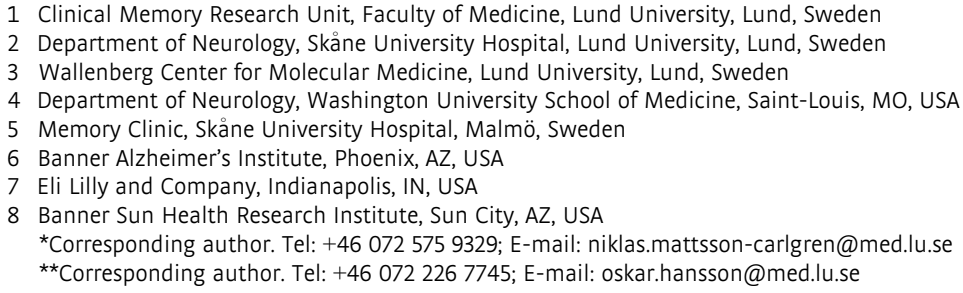


remained statistically associated with plasma P-tau217. Taken together, the data from this observational study support the theory that the first increases in release and phosphorylation of soluble tau measured in plasma are driven by $\mathrm{A} \beta$ aggregation and may appear largely before spread of tau tangles outside of the medial temporal lobe. One possibility is that soluble P-tau217 plays a role in the spread of neocortical tangles, which could motivate trials with treatments targeting soluble phosphorylated tau, to break the link between $A \beta$ and tau aggregation in $A D$.

\section{Results}

\section{Plasma P-tau217 is independently associated with pathology measures of both $A \beta$ and tau}

We first tested associations between antemortem plasma P-tau217 and neuropathological quantification of $\mathrm{A} \beta$ plaques and tau tangles in a cohort with post-mortem data $(N=88)$. We compared regression models with plasma P-tau217 as response variable and different sets of predictors, including I) only $A \beta$ plaque density, II) only tau tangle density, III) both $A \beta$ plaque and tangle densities, and IV) $A \beta$ plaque and tangle densities and their interaction. All models included age and sex as covariates. For the individual models, the tangle only model had slightly higher overall explanatory power $\left(R^{2}=0.47\right)$ than the plaque only model $\left(R^{2}=0.40\right)$ $\left(\Delta \mathrm{AIC}=9\right.$ favoring the tangle only model, but the difference in $R^{2}$ was not significant, tested in a bootstrap procedure, $\Delta R^{2}=0.07$, $95 \% \mathrm{CI}-0.17$ to 0.25$)$. Model comparisons favored including both plaques and tangle densities in an interaction model (Fig 1A, $\Delta \mathrm{AIC}=5-29$ compared to the other models). In the interaction model, both plaque density $(\beta=2.00, P<0.0001$; compared to $\beta=2.95, P<0.0001$ in the plaque only model) and tangle density $(\beta=1.46, P=0.0072$; compared to $\beta=3.29, P<0.0001$ in the tangle only model) and the plaque by tangle interaction $(\beta=1.26$, $P=0.012$ ) were significantly associated with higher plasma Ptau217 levels. Age $(P=0.19)$ and sex $(P=0.59)$ were nonsignificant in the interaction model. Higher plaque density had the strongest association with high plasma P-tau217 among those with higher tangle density (visualized with tertiles of tangles in Fig 1B), and higher tangle density had the strongest association with high plasma P-tau217 among those with higher plaque density (visualized with tertiles of plaques in Fig 1C). For all these analyses, plaque and tangle densities were used as continuous variables, centered, and scaled to $z$-scores. These results supported the hypothesis that plasma P-tau217 is increased by independent effects of both $A \beta$ and tau pathology.

\section{Plasma P-tau217 statistically mediates the effect of $A \beta$ on tau}

We next conducted statistical mediation analyses in the neuropathology cohort (Fig 2). Plasma P-tau217 partly mediated the effect of $A \beta$ plaque density on tau tangle density (but $A \beta$ plaque density retained a significant association with tangle density also in
A Prediction of plasma P-tau217

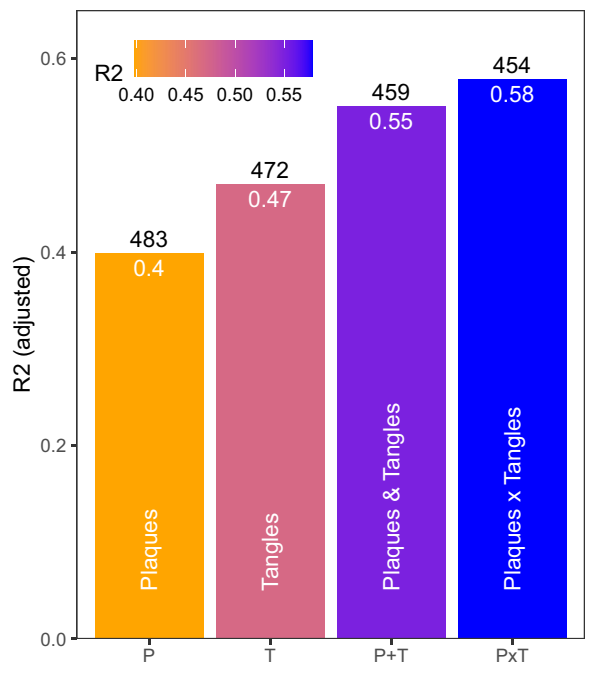

B

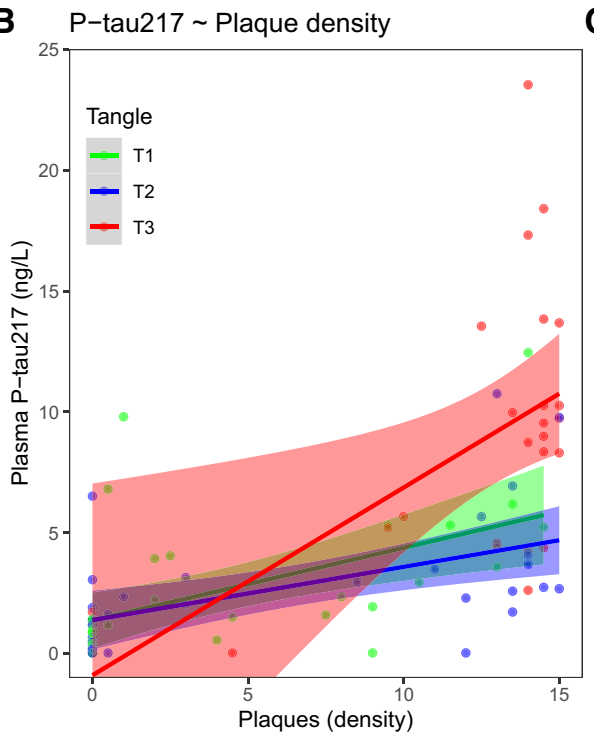

C $\quad$ P-tau217 Tangle density

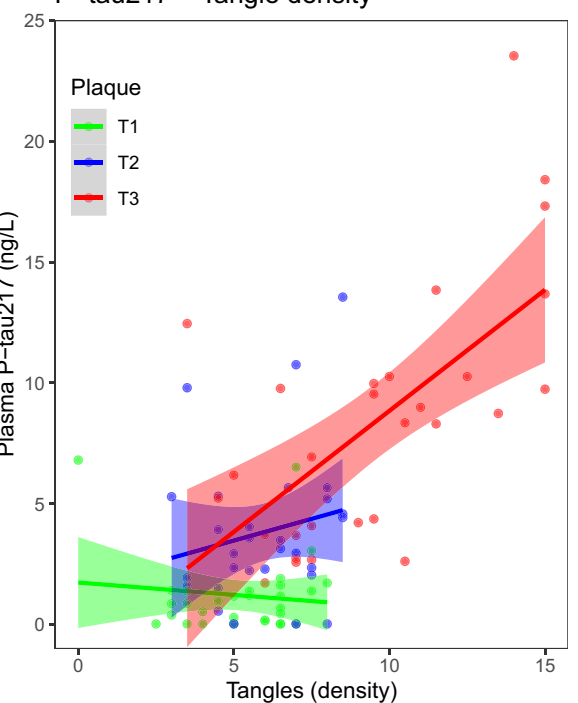

Figure 1. Independent effects of plaque and tangle density on plasma P-tau217.

A $R^{2}$ and AIC for different regression models, using either only amyloid plaque density ("P"), only tangle density ("T"), both plaque and tangle density ("P + T"), or both plaque and tangle density including their interaction term ("PxT"). All models included age and sex as covariates. The panel shows adjusted $R^{2}$, together with AIC (above the bars) for each model. We compared $R^{2}$ between the models using a bootstrap procedure $\left(N=1,000\right.$ iterations), which showed that the $R^{2}$ for the "PxT" model was marginally higher than for the " $\mathrm{P}+\mathrm{T}$ " model $\left(\Delta R^{2}=0.027,95 \% \mathrm{Cl}-0.0034-0.087\right)$ and significantly higher than for the "T" model $\left(\Delta R^{2}=0.12,95 \% \mathrm{Cl}\right.$ 0.04-0.24) and the "P" model $\left(\Delta R^{2}=0.18,95 \% \mathrm{Cl} 0.055-0.31\right)$.

B Associations between plasma P-tau217 and plaque density (stratified by tertiles [T1-3] of tangle density).

C Associations between plasma P-tau217 and tangle density (stratified by tertiles [T1-3] of plaque density).

Data information: Tertiles were chosen to visualize the data in panels (B, C), but the regression models used continuous density data as predictors. The solid lines represent mean effects from regression models. The shaded areas are $95 \%$ confidence intervals for the mean effects. Samples were analyzed in duplicates. 
A
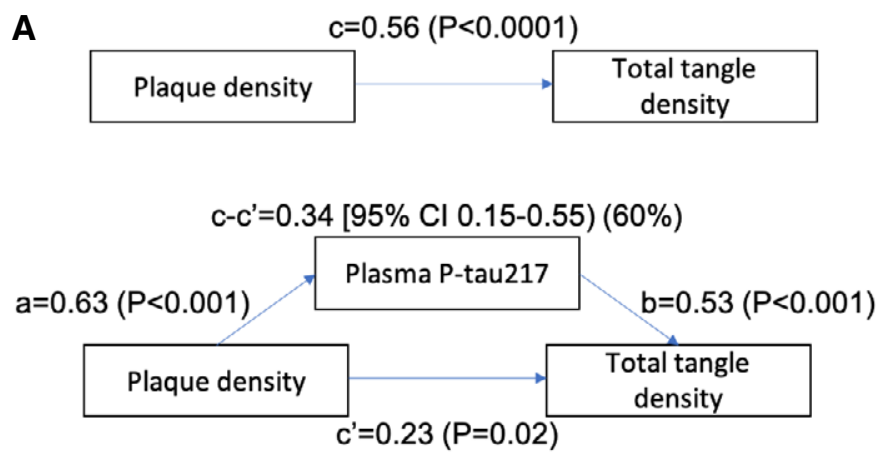

C
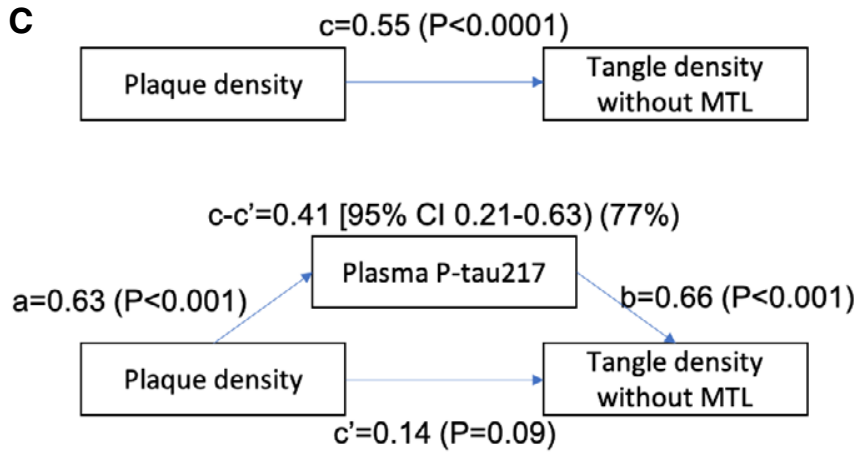

B
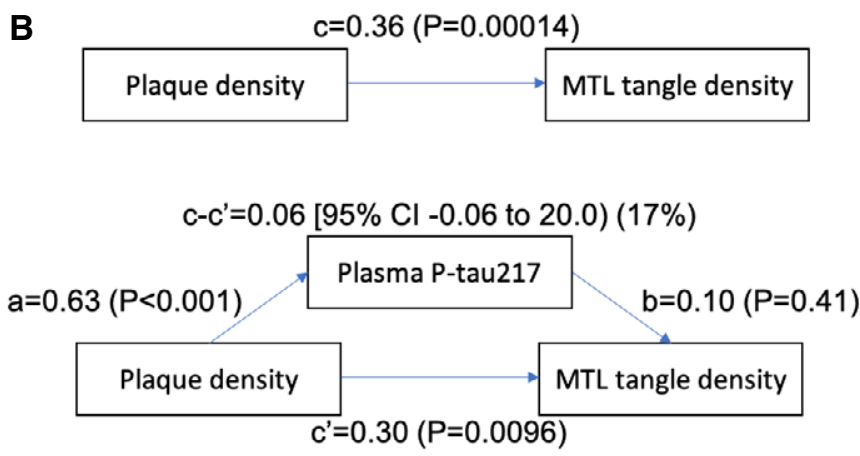

Figure 2. Plasma P-tau217 mediates effects of plaques on tangles.

A-C Three different mediation models are shown, testing plasma P-tau217 as a statistical mediator of plaque density on tangle density. Panel (A) shows the mediation of total tangle density (significant mediation, $60 \%$ of the direct effect of plaque density on tangle density was explained by plasma P-tau217). Panel (B) shows the mediation of medial temporal lobe (MTL, entorhinal cortex and hippocampus) tangle density (no mediation). Panel (C) shows the mediation of tangle density in all regions except the MTL (strongest mediation, 77\%). The $P$-values are from linear regression models used to estimate the mediation effects.

the presence of plasma P-tau217) (60\% mediation, Fig $2 \mathrm{~A})$. In contrast, there was no significant mediation of effects of $A \beta$ plaque density on tau tangle density in the medial temporal lobe (entorhinal cortex and hippocampus) (the direct effect of $A \beta$ plaques on tau tangles was also weaker, Fig $2 \mathrm{~B}$ ). The strongest mediation was seen for total tangle density when removing tangles in the medial temporal lobe $(77 \%$ mediation; the direct effect of $A \beta$ plaques on tangles became non-significant, Fig $2 \mathrm{C}$ ). These results suggest that plasma P-tau217 may partly explain the link between build-up of $A \beta$ and tau pathology, especially for tau pathology that extends over the neocortex, beyond the medial temporal lobe.

\section{No associations between plasma P-tau217 and isolated medial temporal lobe tau}

To study the effects of very subtle tau pathology on plasma Ptau217, we did a subset analysis on the neuropathology cohort participants without tangles in the parietal or frontal lobe (and only minimal tangle pathology in the temporal lobe, allowing score 0.5 , indicating a density halfway between "none" [0] and "sparse" [1])). In this subset $(N=42), A \beta$ plaque density remained significantly associated with plasma P-tau217 $(\beta=1.64, P<0.0001)$ (Fig $3 \mathrm{~A}$ ), but medial temporal lobe tangles were not associated with plasma P-tau217 ( $P=0.40$, Fig $3 \mathrm{~B})$ and there was no association between $A \beta$ plaque density and medial temporal lobe tangle density $(P=0.59$, Fig $3 C)$. These results suggest that $A \beta$ pathology is linked to increased phosphorylation and/or release of tau even in very early stages of $\mathrm{AD}$ (without prominent tangle pathology in the neocortex). In contrast, tau pathology which is contained in the medial temporal lobe without significant neocortical involvement appears to possibly occur independently of $A \beta$ pathology and without altered phosphorylation or release of soluble tau.

\section{No associations between plasma P-tau217 and primary tauopathies (CBD and PSP)}

To test whether plasma P-tau217 was elevated in individuals with a primary non-AD tauopathy, we performed a sensitivity analysis that included nine additional subjects with neuropathology data and a primary neuropathological diagnosis of PSP $(N=7)$ or CBD $(N=2)$ (7 females/2 males; mean age [SD] at death: 87 [8.7] years; mean time between sample collection and death: 1.1 [0.75] years). These were included from the same neuropathology program as the main pathology cohort. Figure 4 shows plasma P-tau217 (Fig 4A), plaque density (Fig 4B), and tangle density (Fig 4C) for these subjects and (for comparison) for subjects with no significant primary pathology, AD, Parkinson's disease, or other primary pathologies. PSP/CBD subjects had low plasma P-tau217 (no difference compared to subjects without significant primary pathology; $P=0.66$, Mann-Whitney U-test). Further, they also had low plaque density (no difference compared to subjects without significant primary pathology; $P=0.13$ ) and a trend to elevated tangle counts 
A P-tau217 Plaques

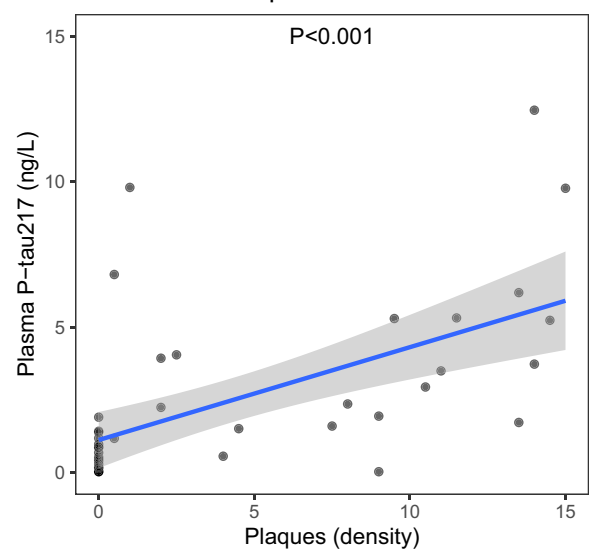

B P-tau217 $\sim$ MTL tangles

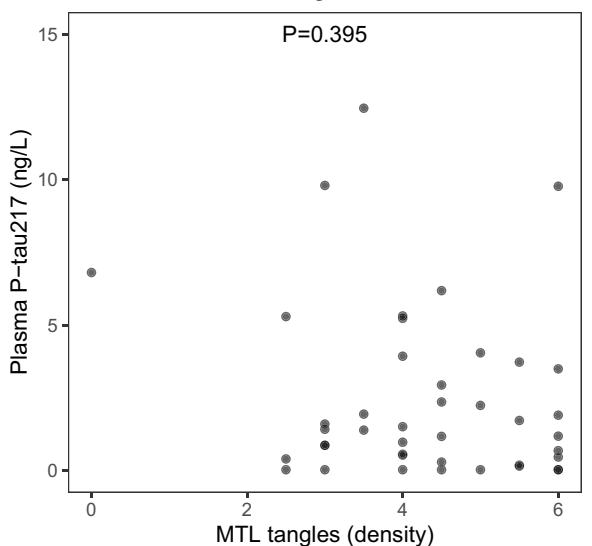

C MTL tangles $\sim$ Plaques

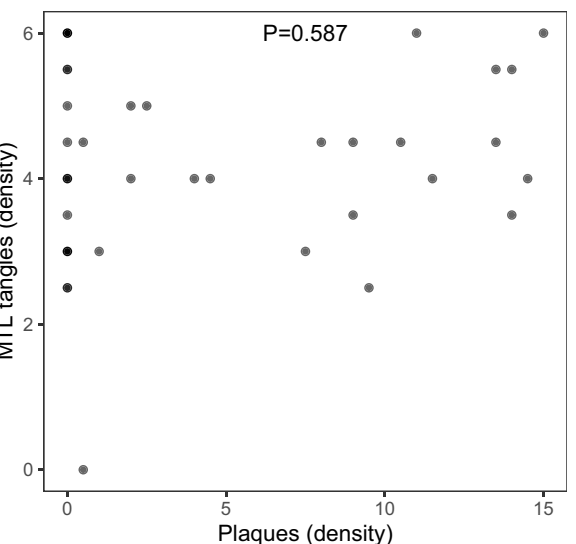

Figure 3. Associations between plasma P-tau217, A $\beta$ plaque density, and medial temporal lobe (MTL) tangle density in people with limited tau pathology.

A-C Individuals in the neuropathology cohort without tangles in the parietal or frontal lobe and no more than minimal tangle load in the temporal lobe were included in this analysis $(N=42)$. MTL tangles were defined as tangles in entorhinal cortex plus hippocampus. Relationships between variables were tested in linear regression models, adjusted for age and sex. In these models, plasma $P$-tau217 was significantly associated with $A \beta$ plaques ( $\beta=1.64, P<0.0001)($ panel $A)$ but not with MTL tangles (panel B). MTL tangles were not associated with A $\beta$ plaques (panel C). The solid line in panel (A) is the mean effect from the regression model, and the shaded area is the $95 \%$ confidence interval of the mean.

A Plasma P-tau217

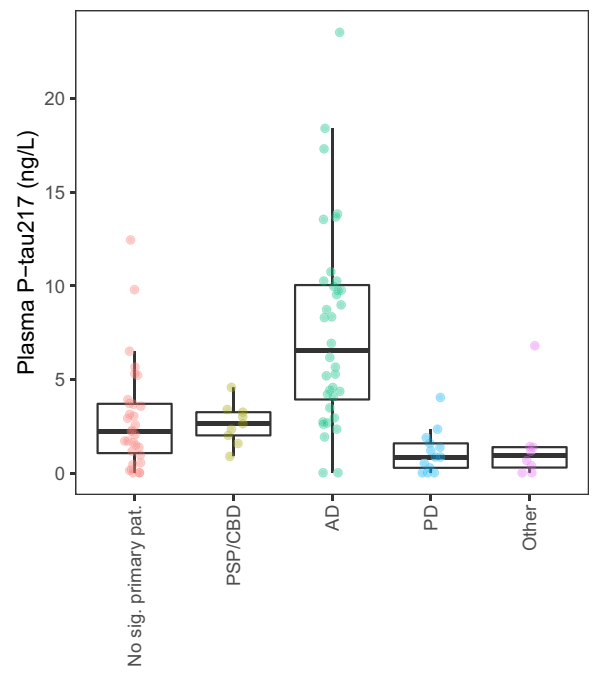

B Plaques

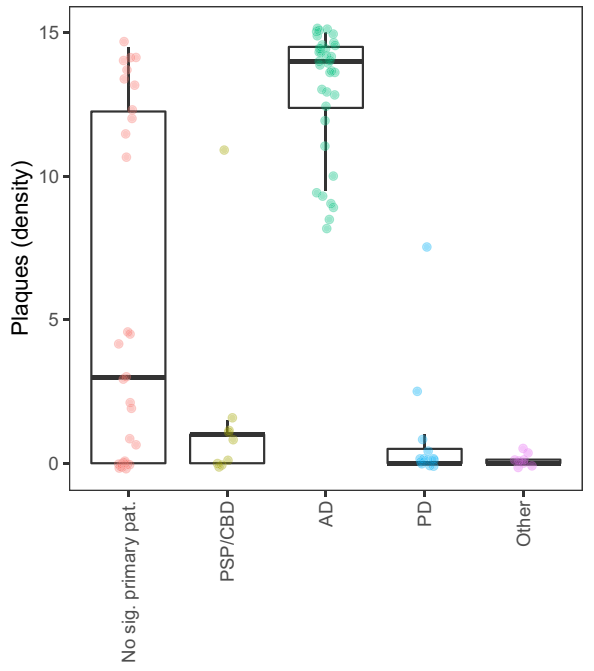

C Tangles

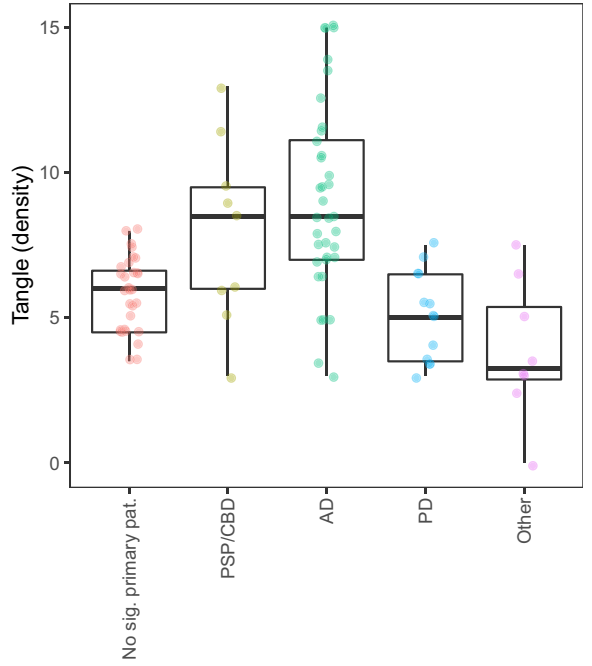

Figure 4. Plasma P-tau217, A $\beta$ plaque density, and tau tangle density by primary neuropathology.

A-C Individuals in the neuropathology cohort were divided into groups of no significant primary pathology $(N=31)$, AD $(N=36)$, PD $(N=13)$, and others $(N=8)$ and analyzed together with $N=9$ additional subjects with primary non-AD tauopathies (PSP/CBD). Data are shown for plasma P-tau217 (panel A), plaque density (panel B), and tangle density (panel C). Plasma P-tau217 was not elevated in PSP/CBD compared to subjects without significant primary pathology. AD, Alzheimer's disease; CBD, corticobasal degeneration; PSP, progressive supranuclear palsy; and PD, Parkinson's disease. The central band of the boxes is medians, and the boxes show interquartile ranges. The whiskers are defined as the smallest (largest) observation greater (less) than or equal to the first (third) quartile minus (plus) 1.5 times the interquartile range.

(higher levels compared to subjects without significant primary pathology, $P=0.068$; no difference compared to $\mathrm{AD}, P=0.37$ ). This suggests that plasma P-tau217 is not necessarily elevated due to tau accumulation in pure non-AD tauopathies, supporting that the increase in plasma P-tau217 is specific to $\mathrm{AD}$ (and may require plaque deposition in addition to tau accumulation).

\section{Plasma P-tau217 is independently associated with PET measures of both $A \beta$ and tau}

We next tested associations between plasma P-tau and measures of aggregated $A \beta$ and tau in vivo in the BioFINDER-2 cohort (see Table 1). We again compared regression models with plasma 
Table 1. Demographics

\begin{tabular}{l|ll} 
& Arizona neuropathology cohort & BioFINDER-2 \\
\hline$N$ & 88 & 426 \\
\hline Men/female & $35 / 53$ & $227 / 199$ \\
\hline Age & $83.7(8.1)$ & $65.7(13.7)$ \\
\hline Diagnostic & 52 NIA-Reagan Not-Low (MMSE & $264 \mathrm{~A} \beta-\mathrm{CU}$ (MMSE \\
group & $26.0[4.3])$ & $29.0[1.2])$ \\
& 36 NIA-Reagan Intermediate-High & $73 \mathrm{~A} \beta+\mathrm{CU}$ (MMSE \\
& (MMSE 20.6 [6.2]) & $28.6[1.4])$ \\
& & $81 \mathrm{~A} \beta+\mathrm{MCl}$ (MMSE \\
& & $26.5[2.0])$ \\
& & $8 \mathrm{~A} \beta+\mathrm{AD}$ dementia \\
& & $(\mathrm{MMSE} 22.3[2.7])$ \\
\hline APOE $\varepsilon 4$ & $\varepsilon 2 / \varepsilon 3 N=8$ & $\varepsilon 2 / \varepsilon 2 N=2$ \\
genotype & $\varepsilon 2 / \varepsilon 4 N=2$ & $\varepsilon 2 / \varepsilon 3 N=24$ \\
& $\varepsilon 3 / \varepsilon 3 N=49$ & $\varepsilon 2 / \varepsilon 4 N=10$ \\
& $\varepsilon 3 / \varepsilon 4 N=26$ & $\varepsilon 3 / \varepsilon 3 N=182$ \\
& $\varepsilon 4 / \varepsilon 4 N=3$ & $\varepsilon 3 / \varepsilon 4 N=180$ \\
& & $\varepsilon 4 / \varepsilon 4 N=28$ \\
\hline
\end{tabular}

Continuous data are mean (standard deviation). Mini-Mental State Examination (MMSE) score is provided for the different diagnostic groups. NIA-Reagan refers to the National Institute on Aging-Reagan Institute criteria for the neuropathological diagnosis of Alzheimer disease (Hyman \& Trojanowski, 1997). CU, cognitively unimpaired. MCl, mild cognitive impairment. AD, Alzheimer’s disease.

P-tau217 as response variable and different sets of predictors, including I) only A $\beta$ PET (using a large cortical region-of-interest [ROI]), II) only tau PET (using a global cortical ROI representing Braak stages I-VI), III) both A $\beta$ PET and tau PET, and IV) A $\beta$ PET and tau PET and their interaction. Model comparison of adjusted $R^{2}$ and AIC favored the model with the interaction term over the other models (Fig $5 \mathrm{~A})$. In the interaction model, both $\mathrm{A} \beta$ PET $(\beta=1.07$, $P<0.0001)$ and tau PET $(\beta=0.44, P<0.0001)$ and the A $\beta$ by tau PET interaction $(\beta=0.31, P<0.0001)$ were significantly associated with greater plasma P-tau217 (A $\beta$ and tau PET were used as continuous variables, centered, and scaled to $z$-scores $)$. Age $(P=0.89)$ and sex $(P=0.91)$ were non-significant predictors in the model. Higher A $\beta$ PET was associated with higher plasma P-tau217 at all levels of tau PET (with strongest association in those with highest tau PET) (Fig 5B). Higher tau PET was associated with higher plasma P-tau217 in those with high (but not in those with low) A $\beta$ PET (Fig 5C) (but we acknowledge that the narrow range of tau PET in the individuals with low A $\beta$ PET makes it more difficult to detect a significant association with plasma P-tau217, compared to in those with high A $\beta$ PET, where the range of tau PET is wider). These results largely agreed with our results from the neuropathology dataset, showing that plasma P-tau217 is independently related to both $A \beta$ and tau build-up.

\section{Plasma P-tau217 correlations with $\mathrm{A} \beta$ and tau PET stratified by $A \beta$ and tau positivity}

The finding that P-tau217 was independently related to both neuropathological densities of plaques and tangles, as well as both $\mathrm{A} \beta$ and tau PET measures, led us to the hypothesis that plasma Ptau217 mainly reflects $A \beta$ pathology during the early stages of the disease (prior to significant neocortical spread of tangles), but is more closely related to tau pathology during the later stages of the disease (when both $A \beta$ and tau pathologies are widespread). To test this hypothesis in vivo, we grouped the BioFINDER-2 participants into three groups: negative for both A $\beta$ PET and tau PET $(N=264)$, A $\beta$ PET-positive but tau PET-negative $(N=104)$, and positive for both $A \beta$ and tau PET $(N=58)$. For this grouping, we used the tau PET uptake in a neocortical ROI corresponding to Braak regions I-IV (Leuzy et al, 2020). No individual in this dataset had negative $A \beta$ PET combined with positive tau PET. Figure 5 (panels D-I) shows correlations between plasma P-tau217 and A $\beta$ PET (Fig 5D-F) and tau PET (Fig 5G-I). There were no correlations between plasma Ptau217 and either $A \beta$ or tau PET in the $A \beta$ - and tau-negative group $(P>0.05$, Fig $5 \mathrm{D}$ and $5 \mathrm{G})$. In those with isolated $A \beta$ positivity, plasma P-tau217 correlated with A $\beta$ PET $(P<0.001$, although the explanatory power of $\mathrm{A} \beta$ on plasma $\mathrm{P}$-tau 217 was modest, $R^{2}=0.10$, Fig 5E) but not tau PET $\left(P=0.92, R^{2}=0\right.$, Fig $\left.5 \mathrm{H}\right)$. In the group positive for both $A \beta$ and tau, plasma P-tau217 correlated with both $\mathrm{A} \beta$ PET $\left(R^{2}=0.14\right.$ for $\mathrm{A} \beta$, Fig $\left.5 \mathrm{~F}\right)$ and tau PET $\left(R^{2}=0.46\right.$ for tau, Fig 5I) but had strongest correlation with tau PET. This supported our hypothesis that plasma P-tau217 reflects both $A \beta$ and tau accumulation in $\mathrm{AD}$, but primarily reflects $\mathrm{A} \beta$ accumulation during the early disease stages (when tau PET is still negative) and

Figure 5. Associations between plasma P-tau217 with A $\beta$ PET and tau PET.

A $\quad R^{2}$ and AIC for different regression models, using either only A $\beta$ PET (“A”), only tau PET ("T(I-VI)"), both A $\beta$ and tau PET (“A + T"), or both A $\beta$ and tau PET including their interaction term ("AxT"). All models included age and sex as covariates. The panel shows adjusted $R^{2}$, together with AIC (above the bars) for each model. We compared $R^{2}$ between the models using a bootstrap procedure ( $N=1,000$ iterations), verifying that the $R^{2}$ for the "AxT" model was higher than for the "A $+\mathrm{T}$ " model $\left(\Delta R^{2}=0.026,95 \% \mathrm{Cl} 0.010-0.058\right)$, the "T(I-VI)" model $\left(\Delta R^{2}=0.17,95 \% \mathrm{Cl} 0.11-0.26\right)$, and the "A" model $\left(\Delta R^{2}=0.14,95 \% \mathrm{Cl}\right.$ 0.060-0.22).

B Associations between plasma P-tau217 and A $\beta$ PET stratified by tertiles (T) of tau PET (T1: SUVR $\leq 1.05$, T2: $1.05<$ SUVR $\leq 1.11$, T3: $1.11<$ SUVR $\leq 3.03$ ).

C Associations between plasma P-tau217 and tau PET stratified by tertiles (T) of A $\beta$ PET (T1: SUVR $\leq 0.468$, T2: $0.468<$ SUVR $\leq 0.579$, T3: 0.579 $<$ SUVR $\leq 1.08$ ).

D-I Associations with P-tau217 across groups of A $\beta$ and tau PET positivity. Cut-points for A $\beta$ PET ( $>0.533$ SUVR) and tau PET ( $>1.36$ SUVR in a ROI corresponding to Braak stages I-IV) were used to define the groups. Associations are shown for global cortical A $\beta$ PET (panels D-F) and global cortical tau PET (corresponding to Braak stages I-VI) (panels $\mathrm{G}-\mathrm{I}$ ) in individuals classified as negative for both $A \beta$ and tau $(N=264$, panels $D$ and $G$ ), positive for $A \beta$ only $(N=104$, panels $E$ and $H)$, or positive for both $A \beta$ and tau ( $N=58$, panels $F$ and I). The plots show $R^{2}$ and $P$-values from linear regression models. No covariates were included in these models, in order to generate $R^{2}$-values for the PET measures alone.

Data information: In panels (B, C), tertiles were chosen to visualize the data, but the regression models used continuous PET uptake as predictors. In panel (C), one outlier was beyond the range of the $\mathrm{x}$-axis (plasma P-tau217 $=17.8 \mathrm{ng} / \mathrm{L}$, tau PET $=3.03 \mathrm{SUVR}, 3^{\text {rd }} \mathrm{A} \beta$ PET tertile). The solid lines represent mean effects from regression models. The shaded areas are $95 \%$ confidence intervals for the mean effects. Samples were analyzed in duplicates. 
A Prediction of plasma P-tau217

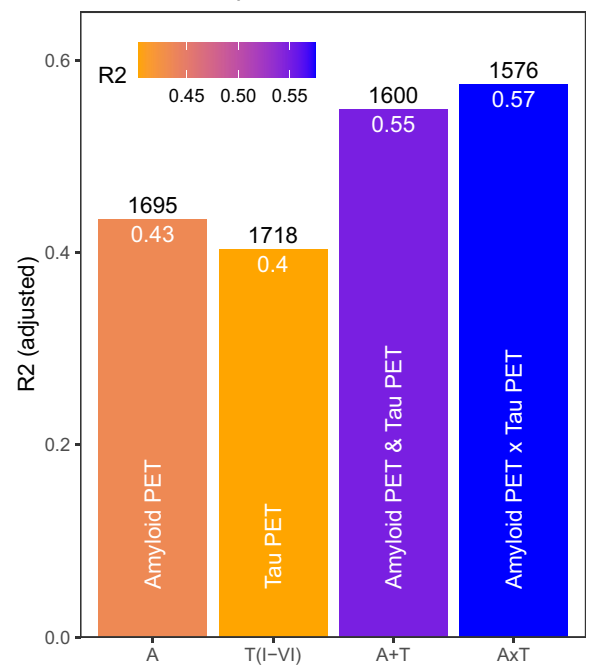

D Amyloid PET (A-T-)

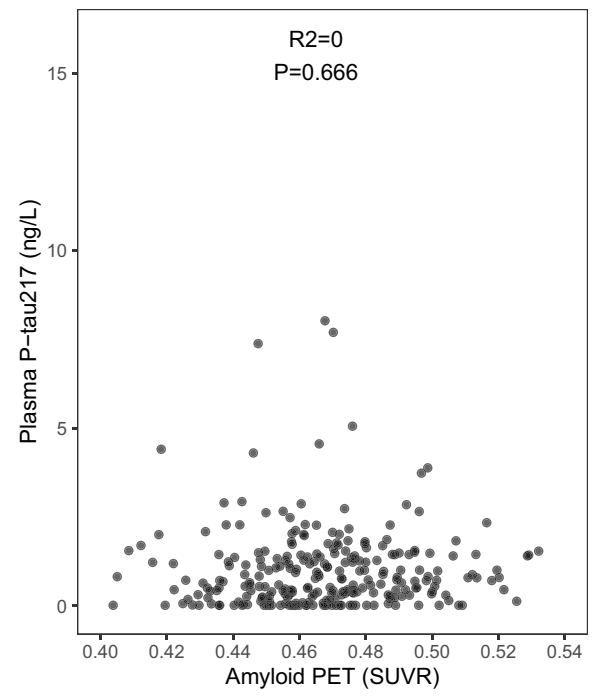

G Tau PET (A-T-)

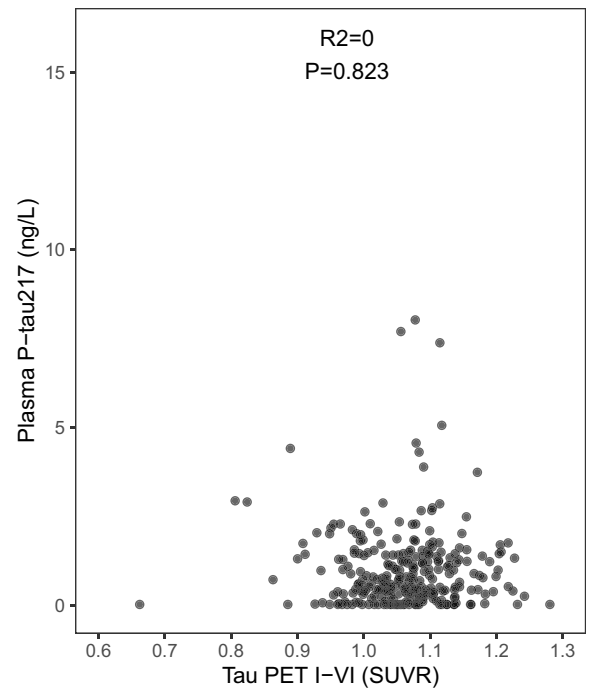

Figure 5.
B $\quad$ P-tau217 Amyloid PET

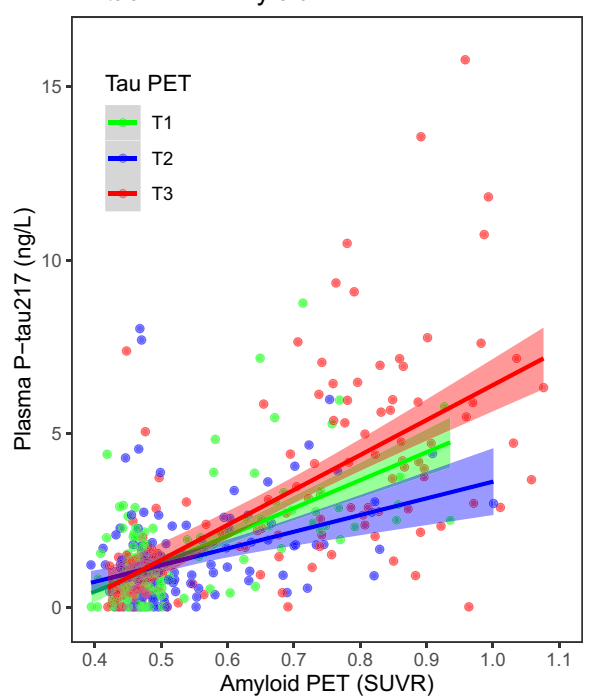

E Amyloid PET (A+T-)

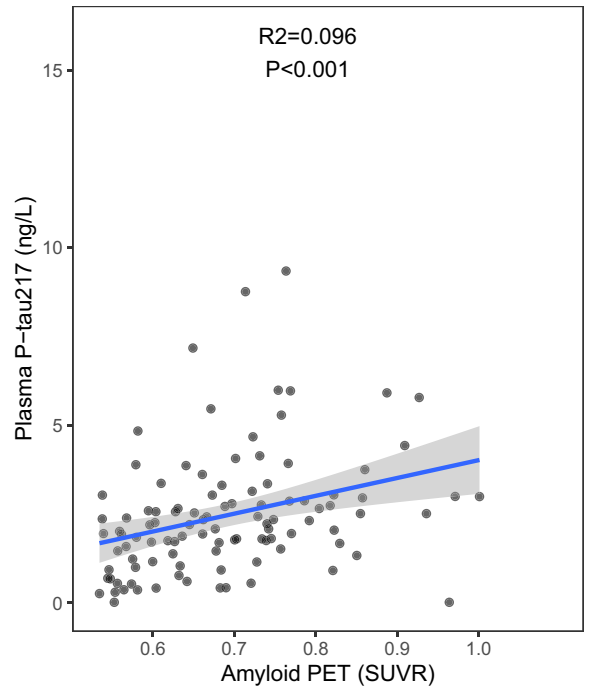

H Tau PET $(\mathrm{A}+\mathrm{T}-)$

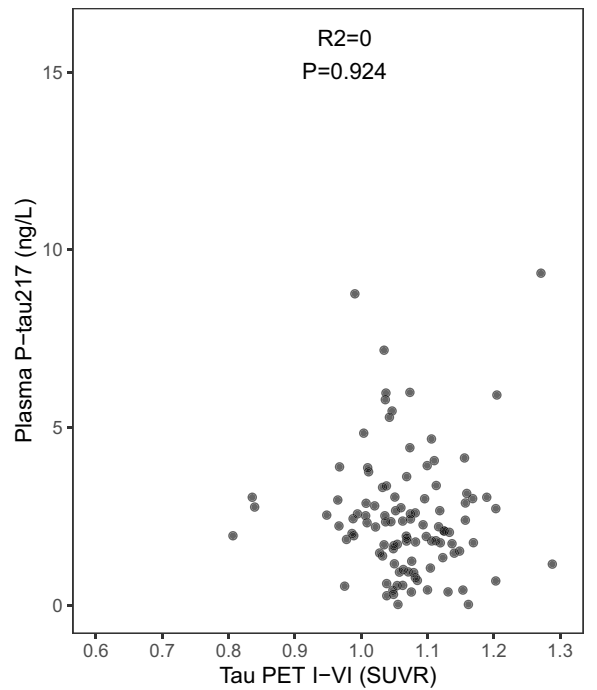

C P-tau217 $\sim$ Tau PET

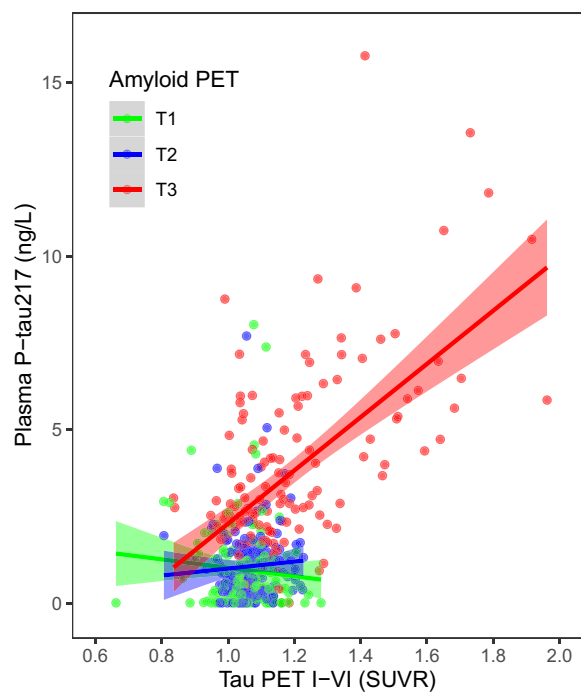

F Amyloid PET $(\mathrm{A}+\mathrm{T}+)$

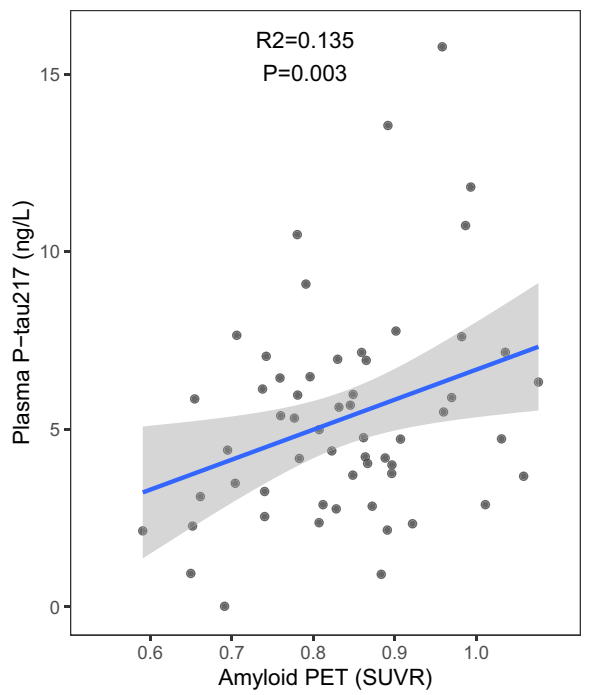

I Tau PET $(\mathrm{A}+\mathrm{T}+)$

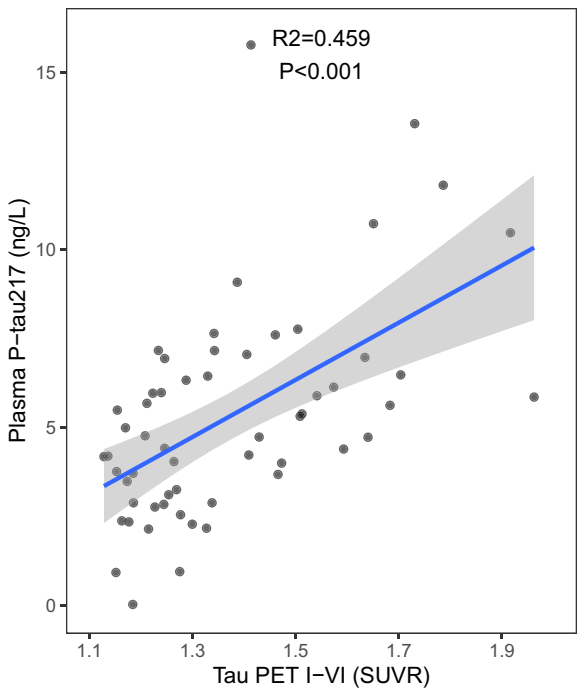


primarily tau accumulation during the later disease stages (when both $A \beta$ and tau PET are positive).

\section{Plasma P-tau217 statistically mediates effects of A $\beta$ PET on tau PET}

We also conducted statistical mediation analyses to test to what degree plasma P-tau217 explained effects of $A \beta$ aggregation on tau uptake in BioFINDER-2. Plasma P-tau217 partly mediated the effect of A $\beta$ PET on global tau PET (63\% mediation, Fig 6A). The mediation was attenuated for tau PET in the medial temporal lobe (entorhinal cortex and hippocampus, $29 \%$, Fig 6B). This is consistent with the hypothesis that the mechanism that links $A \beta$ build-up to tau aggregation involves increased phosphorylation and/or release of soluble tau, and that this is especially important for tau aggregation that occurs outside of the medial temporal lobe (entorhinal cortex and hippocampus).

\section{Discussion}

With recent advances in assay development, it is now possible to measure plasma levels of P-tau217 in clinical research, drug trials, and perhaps also in clinical practice in the future. To best interpret the P-tau217 results, it is important to understand what plasma Ptau217 levels represent. Although proposed frameworks have largely assumed that soluble P-tau best mirrors tau deposition (Jack et al, 2018), we and others have previously noted that CSF P-tau may be increased also as a function of $A \beta$ deposition (Barthélemy et al, 2020b; Mattsson-Carlgren et al, 2020)-i.e., provide an indicator of $A \beta$-related tau pathophysiology that anticipates the development of measurable tau tangle pathology. We therefore hypothesized that plasma P-tau217 levels would be related to both $A \beta$ and tau aggregation. Supporting our hypothesis, we found that both $A \beta$ plaques and tau tangles were independently associated with higher plasma P-tau217 levels, when measured both post-mortem by neuropathological quantification (providing the most sensitive indicator of tau tangle deposition) and in vivo by PET imaging (which may not detect tau tangles until there is more substantial and spatially extensive tangle deposition). $A \beta$ plaques and tau tangles also interacted, so that highest plasma P-tau217 levels were seen in individuals who had high levels of both $A \beta$ plaques and tau tangles. The independent effects of $A \beta$ load and tau tangle load on plasma P-tau217 were significant, with considerable increases in model $R^{2}$ when combining $\mathrm{A} \beta$ and tau predictors compared to when using just one of them alone. Even though the results were mainly similar when using either post-mortem neuropathology examination or in vivo PET imaging to detect tau tangles, we found that plasma P-tau217 was more strongly associated with tau tangles than $\mathrm{A} \beta$ plaques when using neuropathology examination, which was not the case when using PET (compare Fig 1A with Fig 5A). This is probably explained by the fact that tau PET imaging does not reliably detect lower amounts of tau aggregates in the brain, as shown in a recent end-of-life study by Fleischer et al evaluating the diagnostic performance of 18F-flortaucipir (Fleisher et al, 2020). However, even when using neuropathology examination, A $\beta$ pathology had an independent effect on plasma P-tau217 levels, and in cases with more limited tau pathology (mainly restricted to the medial temporal lobe), plasma P-tau217 correlated with A $\beta$ pathology but not tau tangles, although we acknowledge that the methods used for tau quantification in the brain may not be sensitive to the earliest most subtle tau aggregation (Fig 3) (and since the total amount of aggregated tau is low in subjects with tau tangles limited to the medial temporal cortex, it is more difficult to detect an association between tangle load and plasma P-tau217 in those individuals compared to when tangles have also spread into neocortex). These results are congruent with recent studies showing that both blood and CSF P-tau217 are associated with A $\beta$ PET also in tau PETnegative cases (Mattsson-Carlgren et al, 2020; Janelidze, 2021). Together, these results imply that plasma P-tau217 is not associated with tau tangle pathology independent from $A \beta$ pathology, including primary age-related tauopathy (PART, see Fig 3B) (Sperling et al, 2014). However, plasma P-tau217 is associated with $A \beta$ pathology, even during the early stages of the disease (Fig 3A), and it is further strongly associated with more widespread tau tangles in cases with $\mathrm{A} \beta$ pathology (Figs $1 \mathrm{C}$ and $4 \mathrm{C}$ ). More research is needed to understand the molecular and cellular mechanisms behind how early $A \beta$ pathology leads to changes in extracellular P-tau217 levels.
A
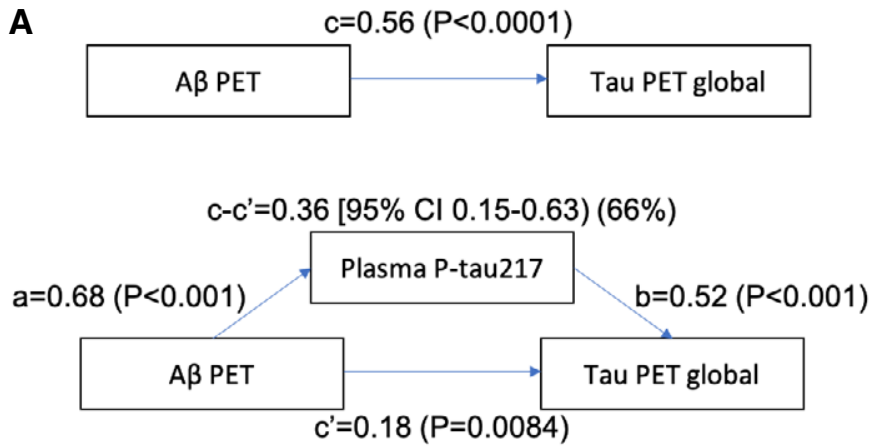

B
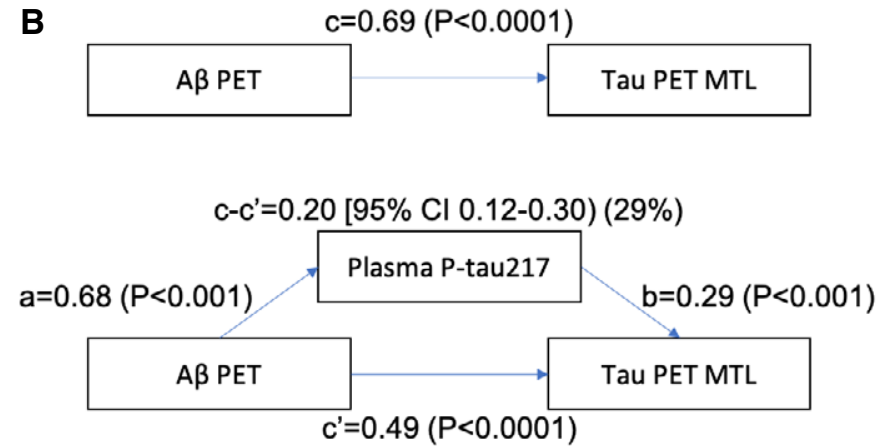

Figure 6. Mediation analyses in BioFINDER-2.

A, B Plasma P-tau217 partly mediated the effect of A PET on tau PET, when using tau PET quantified in Braak stages I-VI (A, "Tau PET global", 66\% of the direct effect of $A \beta$ PET on tau PET was explained by plasma $P$-tau217) or in the medial temporal lobe (B, MTL, entorhinal cortex and hippocampus, $29 \%)$. The $P$-values are from linear regression models used to estimate the mediation effects. 


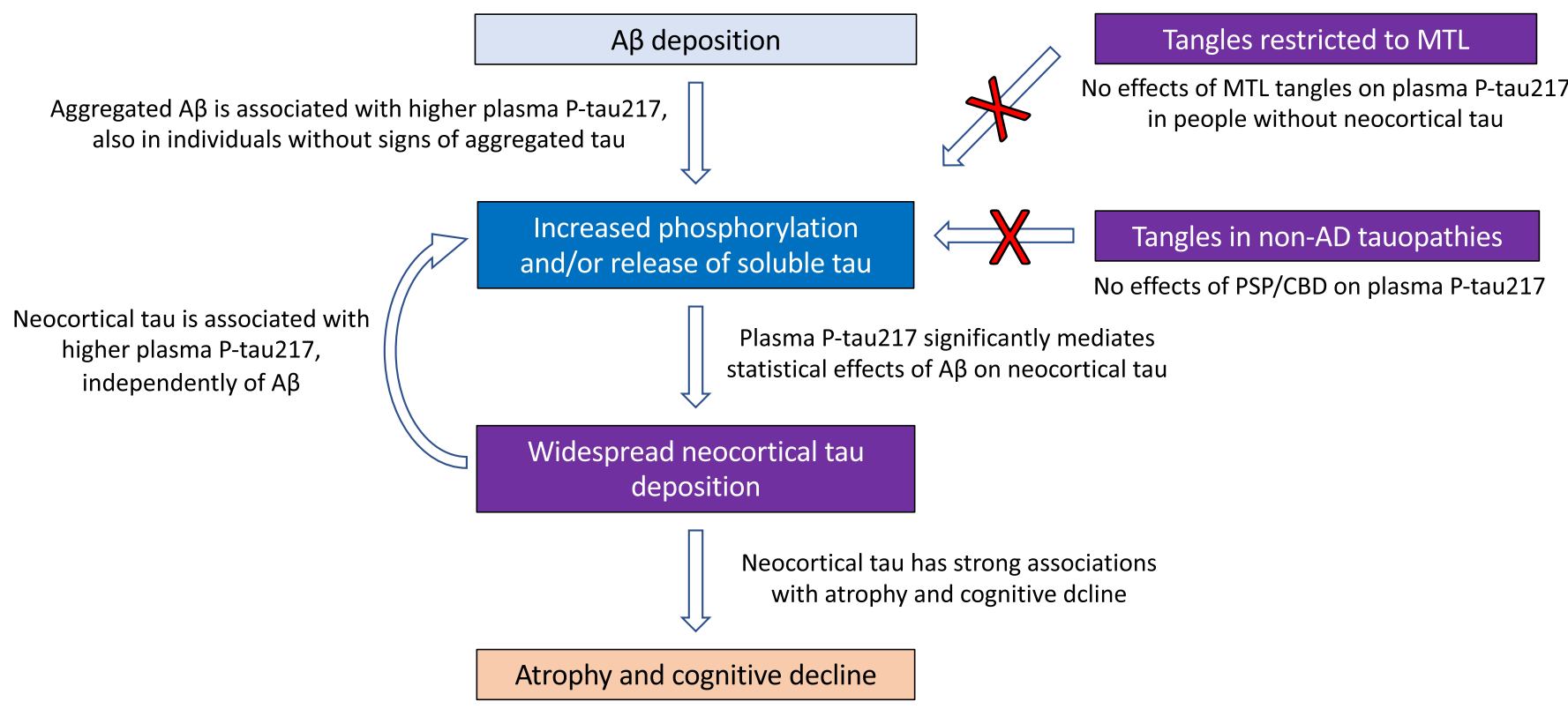

Figure 7. Hypothetical integration of neuropathology, fluid biomarker, and PET imaging findings.

The figure shows hypothetical pathways, supported by our results, which link deposition of $A \beta$ pathology to development of neocortical tangles through increased phosphorylation and/or release of soluble tau. We also include a hypothetical feedback loop, where tangle pathology also may drive increased levels of soluble P-tau, possible due to tau propagation between affected neurons. We also acknowledge that other alternative explanations may be considered for our findings. One possibility is that lack of sensitivity of our methods to accurately quantify aggregated tau makes us unable to detect very early associations between aggregated tau and plasma Ptau217.

Overall, the link between $\mathrm{A} \beta$ pathology and increased plasma Ptau217 is in line with recent results from cell models (Kwak et al, 2020) showing that phosphorylation of tau is increased in the presence of $A \beta$.

Plasma P-tau217 also provided statistical mediation for the effects of $A \beta$ load on tau load in both the neuropathology dataset and the BioFINDER-2 PET imaging dataset. Intriguingly, the mediation effect of P-tau217 was especially strong for neuropathological quantification of tangle burden in the neocortex (excluding tangle count densities in entorhinal cortex and hippocampus, Fig 2C), where plasma P-tau217 explained up to $77 \%$ of the effect of $A \beta$ plaques on tangle burden and the direct effect of $A \beta$ plaques on tangles became non-significant when correcting for P-tau217. The in vivo measures with $A \beta$ and global tau PET showed similar results, with considerable statistical mediation for plasma P-tau217 on global tau PET (66\%), but less mediation (29\%) when using tau PET quantified in the entorhinal cortex or hippocampus. This shows that plasma P-tau217 may be especially important for the spread of tau outside of the medial temporal lobe. Isolated medial temporal lobe tangle pathology (which may even appear in the absence of $A \beta$ pathology, including "PART" (Crary et al, 2014)) appears not to be strongly related to increased phosphorylation and/or secretion of tau.

When grouping the subjects into three hypothetical "disease stages” (Fig 6), we found no correlations between plasma P-tau217 and either A $\beta$ PET or tau PET in those that were negative for both $A \beta$ and tau PET (we consider these individuals to not have AD), significant correlations to A $\beta$ PET (but not tau PET) in those who were within the positive range for $A \beta$ PET only (we consider this an early stage of $\mathrm{AD}$ ), and significant correlations to both $\mathrm{A} \beta$ PET and tau PET (but strongest with tau PET) in those who were positive for both $A \beta$ and tau PET (we consider this a later stage of AD). Taken together, these findings are congruent with the hypothesis that $A \beta$ pathology leads to an increased release of soluble P-tau, which in turn is associated with a spread of tau tangle pathology beyond the medial temporal lobe. Figure 7 shows a hypothetical model which integrates the findings from this study and previous studies on biomarkers and development of $\mathrm{AD}$. However, we note that different sensitivities of the biomarkers to detect underlying pathologies could impact their correlations. One possibility is that plasma P-tau217 may reflect AD-related tau hyperphosphorylation earlier and with higher sensitivity than tau PET. Alternatively, the plasma P-tau217 may initially increase due to increased site phosphorylation at 217 in the $A \beta$ growth stage, and then as soluble total tau concentrations increase later, the P-tau217 increases further, but without specific increases in phosphorylation at site 217 (Barthélemy et al, 2020a, 2020b). Additional valuable information could come from $A \beta$ overproducing mouse models that do not develop tau tangles, to characterize the extent to which $A \beta$ could promote tau secretion even in the absence of subsequent tau tangle deposition (Maia et al, 2015; Mattsson-Carlgren et al, 2020).

Over the last years, there has been an increased interest in antitau treatments for AD (Bittar et al, 2020). One hypothetical possibility raised by our results is that therapies targeting the processes that lead to production of P-tau217 may break the link between build-up of aggregated $A \beta$ and tau and thereby reduce atrophy and cognitive decline in $\mathrm{AD}$ (which are both strongly associated with tau pathology (Mattsson et al, 2019; Ossenkoppele et al, 2019)). At least one tau-directed treatment (using an anti-tau antibody) has been 
reported to reduce CSF levels of P-tau217 (conference presentation by Michael Fresser at AAT-AD/PD 2020). Further drug development may incorporate plasma P-tau217 data in early stages to select drug candidates most likely to have beneficial effects on the biochemistry of tau in the brain in AD.

A limitation of this study is the relatively small dataset in the neuropathology cohort, with a very wide spread of different pathologies. Especially, there were quite few study participants with intermediate plaque counts (most had either very few plaques or substantial plaque pathology). Larger neuropathology datasets would be valuable to validate the findings directly in post-mortem material. We used the large BioFINDER-2 cohort to validate the findings, but we acknowledge that tau PET has limited sensitivity for early stage tau pathology (with isolated tangle pathology in entorhinal cortex and hippocampus). We therefore refrained from doing some of the subgroup analyses in BioFINDER-2 (focusing on MTL tau in individuals with negative global tau). Another limitation is that we used cross-sectional data, and longitudinal data for multiple modalities are needed to strengthen hypotheses about causal relationships between the biomarkers.

In summary, plasma P-tau217 is independently associated with both $A \beta$ plaques and tau tangles, including associations with $A \beta$ pathology even in cases with restricted tau tangle pathology and strong associations with tau tangles in A $\beta$-positive cases.

\section{Materials and Methods}

\section{Participants}

Study participants were from two cohorts. First, we included subjects from a biomarker-neuropathology cohort study (the Arizona Study of Aging and Neurodegenerative Disorders/Brain and Body Donation Program), also described previously (Palmqvist et al, 2020). Second, we included subjects from the prospective cohort study BioFINDER-2 (clinical trials: NCT03174938), explained previously (Palmqvist et al, 2020). From BioFINDER-2, we included all available cognitively unimpaired controls, $A \beta+$ mild cognitive impairment $(\mathrm{MCI}$ ), and $\mathrm{A} \beta+\mathrm{AD}$ dementia patients (where $\mathrm{A} \beta+$ was determined by A $\beta$ PET, explained below) with $A \beta$ and tau PET data. Study demographics are summarized in Table 1 . The data used for this paper partly overlap with data in a previous publication in diagnostic performance of plasma P-tau217 (Palmqvist et al, 2020) (but the BioFINDER-2 sample used here is more restricted, since both $A \beta$ and tau PET were required for inclusion in this study). Informed consent was obtained from all study participants.

\section{Fluid biomarkers}

Plasma P-tau217 was measured using immunoassays at Lilly Research Laboratories, as described before (Palmqvist et al, 2020). In the Arizona Study of Aging and Neurodegenerative Disorders/ Brain and Body Donation Program, the mean (standard deviation) time between plasma collection and death was 1.2 (0.8) years. In BioFINDER-2, the mean time between plasma collection and PET imaging was $0.16(0.22)$ years. Plasma P-tau217 data were not available to assessors of neuropathology or neuroimaging (minimizing subjective bias).

\section{Neuropathology}

We included subjects from the biomarker-neuropathology cohort with or without neuropathological evidence of $\mathrm{AD}$. $\mathrm{A} \beta$ plaque and neurofibrillary tangles were graded at standard sites in frontal, temporal, parietal, occipital cortices, the hippocampal CA1 region, and entorhinal/transentorhinal cortex, using $80-\mu \mathrm{m}$ sections (Palmqvist et al, 2020). Plaque densities (including cored, neuritic, and diffuse plaques together) were derived using the Campbell-Switzer staining. Neurofibrillary tangle densities were derived using three staining methods (thioflavin S, Campbell-Switzer, and Gallyas) on the same sections. The CERAD templates (Mirra et al, 1991) were used to obtain semi-quantitative regional scores for $A \beta$ and tangle pathology, which were summed to total plaque and tangle scores (range 0-15). All histopathological scoring was blinded to clinical or neuropathological diagnosis (see Table EV1 for details) and to biomarker levels.

\section{PET imaging}

The procedures of tau PET (using R0948 labeled with radioactive fluorine $\left[{ }^{18} \mathrm{~F}\right]$ ) and $\mathrm{A} \beta$ PET (using flutemetamol labeled with ${ }^{18} \mathrm{~F}$ ) have been described previously (Palmqvist et al, 2020). A $\beta$ PET was sampled in a global cortical ROI. When used as a dichotomous variable, A $\beta$ PET $>0.533$ SUVR was defined as a positive scan (defined previously using mixture modeling (Palmqvist et al, 2020)). Tau PET was sampled in different ROIs, corresponding to different Braak regions. For most analyses, we used a large composite corresponding to Braak regions I-VI, in order to comprehensively capture AD-related brain tau pathology. For some analysis, we used a dichotomous variable for neocortical tau PET positivity, using tau PET uptake in a more limited ROI (corresponding to Braak stages I-IV, where tau PET $>1.36$ SUVR was defined as a positive scan (Leuzy et al, 2020)).

\section{Statistics}

Associations between plasma P-tau217 and measures of A $\beta$ and tau were tested in linear regression models, adjusted for age and sex. For models in the neuropathology cohort, we also evaluated models adjusted for the time between plasma sampling and death. This covariate was never significant and did not alter the effects of the other predictors and was therefore not included in the final models. Different regression models were compared on the same subjects and outcome, with different sets of predictors (including only $A \beta$ data, only tau data, or both types of data, with or without an interaction term). Models were compared using $R^{2}$ and Akaike information criterion (AIC, where a lower value indicates a better fit, corrected for model complexity). Assumptions of regression models were assessed by inspection of standard diagnostic plots. Bootstrap procedures $\left(N=1,000\right.$ iterations) were used to compare $R^{2}$ between models. Mediation analyses were done to test the relationship between A $\beta$ load, tau load, and plasma P-tau217 (using bootstrapped estimates of mediation effects). Statistics were done in $R$ (v 4.0.2).

\section{Data availability}

Anonymized data will be shared by request from a qualified academic investigator and as long as data transfer is in agreement 


\section{The paper explained}

Problem

Alzheimer's disease (AD) is characterized by the accumulation of $\beta$ amyloid and tau in the brain. Plasma levels of tau phosphorylated at threonine 217 (P-tau217) are increased in patients with AD, but it is not clear which pathophysiological process drives this biomarker change.

\section{Results}

Using a patient cohort with neuropathological quantification of brain pathologies and a cohort with in vivo positron emission tomography (PET) of $\beta$-amyloid and tau, we show that plasma P-tau217 had independent relationships with both $\beta$-amyloid and tau aggregation. Specifically, plasma P-tau217 appeared to be associated with $\beta$ amyloid also in individuals without signs of widespread neocortical tau pathology and mediated the association between aggregated $\beta$ amyloid and aggregated tau. In patients with both $\beta$-amyloid aggregation and neocortical tau pathology, plasma P-tau217 was strongly associated with neocortical tau. In patients with non-AD tauopathies (without $\beta$-amyloid pathology), there was no increase in plasma Ptau217.

Impact

Our study shows that accumulation of $\beta$-amyloid pathology may induce changes in phosphorylation and release of soluble tau, resulting in increased plasma P-tau217 before tau accumulation spreads in the neocortex. Later in the AD process, as tau aggregation spreads, plasma P-tau217 reflects the widespread accumulation of neocortical tau. Soluble phosphorylated tau may be investigated further to understand the pathobiological mechanisms of $A D$.

with EU legislation on the general data protection regulation and decisions by the Ethical Review Board of Sweden and Region Skåne, which should be regulated in a material transfer agreement.

Expanded View for this article is available online.

\section{Acknowledgements}

Work at the authors' research center was supported by the Swedish Research Council (2016-00906), the Knut and Alice Wallenberg Foundation (2017-0383 and WCMM Fellowship for NMC 2019-2022), the Medical Faculty at Lund University and Region Skåne (WCMM Fellowship for NMC 2019-2022), the Marianne and Marcus Wallenberg Foundation (2015.0125), the Strategic Research Area MultiPark (Multidisciplinary Research in Parkinson's disease) at Lund University, the Swedish Alzheimer Foundation (AF-939932), the Swedish Brain Foundation (FO2019-0326, FO2020-0275), the Parkinson Foundation of Sweden (1280/20), the Skåne University Hospital Foundation (2020-0000028), Regionalt Forskningsstöd (2020-0314), the Konung Gustaf V:s och Drottning Victorias Frimurarestiftelse (2020 NMC), the Bundy Academy (Stora Priset 2020 NMC), and the Swedish federal government under the ALF agreement (2018Projekt0279, 2018-Projekt0054). The Brain and Body Donation Program has been supported by the National Institute of Neurological Disorders and Stroke (U24 NS072026 National Brain and Tissue Resource for Parkinson's Disease and Related Disorders), the National Institute on Aging (P30 AG19610 Arizona Alzheimer's Disease Core Center), the Arizona Department of Health Services (contract 211002, Arizona Alzheimer's Research Center), the Arizona Biomedical Research Commission (contracts 4001, 0011, 05-901, 1001), the Arizona Department of Health Services (Grant No. CTR040636), and the Michael J. Fox Foundation for Parkinson's Research.

\section{Author contributions}

Study design: NMC and OH. Data acquisition: SJ, RS, ES, SP, GES, TGB, EMR. Biochemical analyses: SJ, JLD. Neuroimaging analyses: RS. Funding acquisition: NMC, OH, EMR, TCB. Statistical analyses: NMC. Writing of first draft: NMC. Critical review of the manuscript: NMC, SJ, RJB, RS, ES, GES, EMR, SP, JLD, TGB, $\mathrm{OH}$. Supervision: $\mathrm{OH}$.

\section{Conflict of interest}

$\mathrm{OH}$ has acquired research support (for the institution) from AVID Radiopharmaceuticals, Biogen, Eli Lilly, Eisai, GE Healthcare, Pfizer, and Roche. In the past 2 years, he has received consultancy/speaker fees from AC Immune, Alzpath, Biogen, Cerveau, and Roche. NMC, ES, SP, SJ, and RS have no disclosures. TCB has had research support from the National Institute on Aging, Michael J Fox Foundation for Parkinson's Research, and the State of Arizona and, in the past 2 years, has received consultancy and/or speaker fees from Prothena Biosciences and Vivid Genomics. JLD is an employee of Eli Lilly and Company. Remaining co-authors report no disclosures.

\section{For more information}

See biofinder.se for more information about the study and cohort.

\section{References}

Barthélemy NR, Horie K, Sato C, Bateman RJ (2020a) Blood plasma phosphorylated-tau isoforms track CNS change in Alzheimer's disease. J Exp Med 217: e20200861

Barthélemy NR, Li Y, Joseph-Mathurin N, Gordon BA, Hassenstab J, Benzinger TLS, Buckles V, Fagan AM, Perrin RJ, Goate AM et al (2020b) A soluble phosphorylated tau signature links tau, amyloid and the evolution of stages of dominantly inherited Alzheimer's disease. Nat Med 26: 398-407

Bittar A, Bhatt N, Kayed R (2020) Advances and considerations in AD tautargeted immunotherapy. Neurobiol Dis 134: 104707

Crary JF, Trojanowski JQ, Schneider JA, Abisambra JF, Abner EL, Alafuzoff I, Arnold SE, Attems J, Beach TG, Bigio EH et al (2014) Primary age-related tauopathy (PART): a common pathology associated with human aging. Acta Neuropathol 128: $755-766$

Fleisher AS, Pontecorvo MJ, Devous MD, Lu M, Arora AK, Truocchio SP, Aldea P, Flitter M, Locascio T, Devine M et al (2020) Positron emission tomography imaging With [18F]flortaucipir and postmortem assessment of Alzheimer disease neuropathologic changes. JAMA Neurol 77: 829-839

Hampel H, O'Bryant SE, Molinuevo JL, Zetterberg H, Masters CL, Lista S, Kiddle SJ, Batrla R, Blennow K (2018) Blood-based biomarkers for Alzheimer disease: mapping the road to the clinic. Nat Reu Neurol 14: 639-652

Hyman BT, Trojanowski JQ (1997) Consensus recommendations for the postmortem diagnosis of Alzheimer disease from the National Institute on Aging and the Reagan Institute Working Group on diagnostic criteria for the neuropathological assessment of Alzheimer disease. J Neuropathol Exp Neurol 56: 1095-1097

Jack CR, Bennett DA, Blennow K, Carrillo MC, Dunn B, Haeberlein SB, Holtzman DM, Jagust W, Jessen F, Karlawish J et al (2018) NIA-AA research framework: toward a biological definition of Alzheimer's disease. Alzheimers Dement 14: 535-562

Janelidze S (2021) Associations of plasma Phospho-Tau217 levels with tau positron emission tomography in early Alzheimer disease. JAMA Neurol 78 $149-156$

Janelidze S, Mattsson N, Palmqvist S, Smith R, Beach TG, Serrano GE, Chai X, Proctor NK, Eichenlaub U, Zetterberg $\mathrm{H}$ et al (2020) Plasma P-tau181 in 
Alzheimer's disease: relationship to other biomarkers, differential diagnosis, neuropathology and longitudinal progression to Alzheimer's dementia. Nat Med 26: 379-386

Karikari TK, Pascoal TA, Ashton NJ, Janelidze S, Benedet AL, Rodriguez JL, Chamoun M, Savard M, Kang MS, Therriault J et al (2020) Blood phosphorylated tau 181 as a biomarker for Alzheimer's disease: a diagnostic performance and prediction modelling study using data from four prospective cohorts. Lancet Neurol 19: 422-433

Kwak SS, Washicosky KJ, Brand E, von Maydell D, Aronson J, Kim S, Capen DE, Cetinbas M, Sadreyev R, Ning $S$ et al (2020) Amyloid- $\beta 42 / 40$ ratio drives tau pathology in 3D human neural cell culture models of Alzheimer's disease. Nat Commun 11: 1377

Leuzy A, Smith R, Ossenkoppele R, Santillo A, Borroni E, Klein G, Ohlsson T, Jögi J, Palmqvist S, Mattsson-Carlgren $N$ et al (2020) Diagnostic performance of RO948 $\mathrm{F} 18$ tau positron emission tomography in the differentiation of alzheimer disease from other neurodegenerative disorders. JAMA Neurol 77: 955

Maia LF, Kaeser SA, Reichwald J, Lambert M, Obermüller U, Schelle J, Odenthal J, Martus P, Staufenbiel M, Jucker M (2015) Increased CSF A $\beta$ during the very early phase of cerebral $A \beta$ deposition in mouse models. EMBO Mol Med 7: 895-903

Mattsson N, Insel PS, Donohue M, Jögi J, Ossenkoppele R, Olsson T, Schöll M, Smith R, Hansson O (2019) Predicting diagnosis and cognition with 18FAV-1451 tau PET and structural MRI in Alzheimer's disease. Alzheimers Dement 15: $570-580$

Mattsson-Carlgren N, Andersson E, Janelidze S, Ossenkoppele R, Insel P, Strandberg O, Zetterberg H, Rosen HJ, Rabinovici G, Chai X et al (2020) A $\beta$ deposition is associated with increases in soluble and phosphorylated tau that precede a positive Tau PET in Alzheimer's disease. Sci Adu 6: eaaz2387
Mirra SS, Heyman A, McKeel D, Sumi SM, Crain BJ, Brownlee LM, Vogel FS, Hughes JP, van Belle G, Berg L (1991) The Consortium to establish a registry for Alzheimer's Disease (CERAD). Part II. standardization of the neuropathologic assessment of Alzheimer's disease. Neurology 41: $479-486$

Olsson B, Lautner R, Andreasson U, Öhrfelt A, Portelius E, Bjerke M, Hölttä M, Rosén C, Olsson C, Strobel G et al (2016) CSF and blood biomarkers for the diagnosis of Alzheimer's disease: a systematic review and metaanalysis. Lancet Neurol 15: 673-684

Ossenkoppele R, Smith R, Ohlsson T, Strandberg O, Mattsson N, Insel PS, Palmqvist S, Hansson O (2019) Associations between tau, $A \beta$, and cortical thickness with cognition in Alzheimer disease. Neurology 92: e601-e612

Palmqvist S, Janelidze S, Quiroz YT, Zetterberg H, Lopera F, Stomrud E, Su Yi, Chen Y, Serrano GE, Leuzy A et al (2020) Discriminative accuracy of plasma phospho-tau217 for Alzheimer disease vs other neurodegenerative disorders. JAMA 324: 772

Sperling R, Mormino E, Johnson $\mathrm{K}$ (2014) The evolution of preclinical Alzheimer's disease: implications for prevention trials. Neuron 84: 608-622 Suárez-Calvet M, Karikari TK, Ashton NJ, Lantero Rodríguez J, Milà-Alomà M, Gispert JD, Salvadó G, Minguillon C, Fauria K, Shekari M et al (2020) Novel tau biomarkers phosphorylated at T181, T217 or T231 rise in the initial stages of the preclinical Alzheimer's continuum when only subtle changes in A $\beta$ pathology are detected. EMBO Mol Med 12: e12921

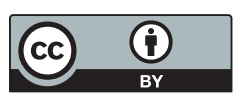

License: This is an open access article under the terms of the Creative Commons Attribution License, which permits use, distribution and reproduction in any medium, provided the original work is properly cited. 